\title{
Clinical features and imaging manifestations of retinoblastoma with hepatic metastasis
}

\author{
Huali $\mathrm{Gu}^{1}$, Dongsheng Huang ${ }^{1}$, Zhuo Wang ${ }^{1}$, Yi Zhang ${ }^{1}$, ling Zhang ${ }^{1}$, Hui-Min Hu${ }^{1}$, Pinwei \\ Zhang ${ }^{1}$, Yan Zhou ${ }^{1}$, You Yi ${ }^{1}$, and Siyu Han ${ }^{1}$ \\ ${ }^{1}$ Beijing Tongren Hospital
}

December 30, 2020

\begin{abstract}
Background: Hepatic metastasis from retinoblastoma (RB) is rare. We evaluated clinical features, imaging manifestations, treatment, and prognosis in these patients. Procedure: Clinical data of five patients diagnosed with hepatic metastases of RB at the Department of Pediatrics in Beijing Tongren Hospital between January 2009 and January 2019 were analyzed retrospectively. Results: Two patients had bilateral lesions, while three had unilateral lesions. Among the seven eyes with lesions, six and one were classified as stage E and C, respectively (International Integrated Reporting Council staging). On computed tomography (CT), low-density foci were observed (three, multiple foci and two, single foci). After chemotherapy, tumor regression was observed in four patients, while there was no response in one patient. Three patients who underwent enucleation were at high risk for extensive choroidal invasion. All patients had severe disease with multiple system involvement, including central nervous system (CNS) and bone metastases. Neuron-specific enolase (NSE) and lactate dehydrogenase (LDH) levels were significantly elevated in all patients; after treatment, they decreased in four patients and remained unchanged in one patient with end-stage disease. Two patients died, with survival durations of 1 and 3 months following the diagnosis of hepatic metastasis. Three patients survived and continued treatment. Conclusion: Hepatic metastasis from RB is rare and usually occurs with CNS and bone metastases. On CT, hepatic foci could be indicated by low-density lesions with calcification. Chemotherapy could be effective for hepatic metastases. The prognosis of these patients is poor; however, hepatic metastasis is not a direct cause of death.
\end{abstract}

\section{Introduction}

Retinoblastoma (RB) is the most common intraocular malignancy in infancy and childhood, with a reported incidence of one per 15,000-20,000 live births. ${ }^{1}$ RB accounts for $3 \%$ of all pediatric cancers. ${ }^{2}$ The overall $^{2}$ survival rate of RB among children diagnosed early at the localized intraocular phase was reported to exceed $95 \%^{3}, 4$; however, delayed diagnosis and treatment, which are common in developing countries, may lead to extraocular metastasis, visual loss, and death. The sites of RB distant metastases include the central nervous system (CNS), bone, bone marrow, lymph nodes, and other organs ${ }^{5-9}$; hepatic metastases are rare. The objective of this study was to evaluate and summarize the clinical features, imaging manifestations, treatment, and prognosis of patients with RB having hepatic metastases.

\section{Methods}

A total of 2,200 patients with RB were admitted at the Department of Pediatrics in Beijing Tongren Hospital, Beijing, China, between January 2009 and January 2019. This hospital is one of the largest and best tertiary eye centers in China. Among the patients with RB, five patients with hepatic metastases were eligible for this study, and their clinical data were analyzed retrospectively. The study protocol was approved by the Medical Ethics Committee of the Beijing Tongren Hospital, and written informed consent was obtained from the patients or guardians (in the case of minors) for the publication of this report and associated images. Serum 
biochemistry, neuron-specific enolase (NSE), alpha fetoprotein (AFP), bone marrow cytology, cerebrospinal fluid cytology, superficial lymph node ultrasound, abdominal computed tomography (CT), and contrastenhanced magnetic resonance imaging (MRI) of the orbits were performed on all patients. Positron emission tomography (PET)-CT was recommended for all five patients; however, two patients declined for financial reasons.

Multi-drug combination chemotherapy including vincristine, etoposide, carboplatin/cisplatin, cyclophosphaminde/isfosfamide, and topotecan was administered. ${ }^{8}$ Intrathecal injection of methotrexate, cytosine arabinoside, and dexamethasone was administered when the CNS was involved. ${ }^{10}$

Abdominal CT and contrast-enhanced MRI of the orbit were performed after every two or three chemotherapy cycles. Radiotherapy after chemotherapy and autologous peripheral blood stem cell transplantation (APBSCT) were scheduled. Adverse reactions to radiotherapy and chemotherapy were evaluated according to the Common Terminology Criteria for Adverse Events guidelines. ${ }^{11}$

\section{Results}

Of the enrolled patients, four were male, and one was female. One patient had hepatic metastases at the time of first RB diagnosis, and four were diagnosed with hepatic metastases at the time of relapse or disease progression. The interval between the first symptom and hepatic metastasis ranged from 1-41 months. Three patients had unilateral tumors (two left and one right), while two had bilateral tumors. Using the International Integrated Reporting Council staging, of the seven enrolled eyes, six were classified as stage E, and one was classified as stage C. Detailed clinical characteristics are shown in Table 1.

The hepatic lesions were mainly observed on abdominal CT. Three patients had multiple lesions, while two had single lesions. All lesions were low-density foci. One had significant calcification, and four had no calcification. Mild enhancement was seen in two patients, and no enhancement seen in the other three. After chemotherapy, tumor regression was observed in four patients, while no response was seen in one patient. Representative CT results are shown in Fig. 1 and Fig. 2.

All the administered treatments are shown in Table 2. Though scheduled, no patient underwent radiotherapy and APBSCT. Two patients died, and in one case, the tumor progressed before radiotherapy and stem cell collection. The other two patients are still receiving chemotherapy; radiotherapy and APBSCT will be performed after they have achieved complete remission. Three patients who underwent enucleation had high-risk pathological factors for extensive choroidal invasion. Detailed pathological examination results are shown in Table 3.

Complications of chemo-radiotherapy were evaluated; headache, vomiting, fever, and myelosuppression were common and disappeared after treatment. Neither hemorrhagic cystitis nor peripheral nervous system damage was found.

Results of the laboratory investigations are shown in Table 4. Serum NSE and LDH levels increased significantly in all the patients. After treatment, NSE levels decreased in four patients and were unchanged in one. Liver metabolism indexes and AFP levels were within the normal range. All patients were followed until October 31, 2020 or until death. Two patients died, while three survived. The overall survival durations of the two deceased patients after the diagnosis of hepatic metastasis were 1 month and 3 months; one of them experienced remission after chemotherapy, but died from severe infection in the myelosuppression stage, while the other died of multiple organ failure due to tumor progression. The three surviving patients underwent follow-up at 6,18, and 3 months, respectively. After the diagnosis of hepatic metastasis; two had partial remission, and one experienced tumor progression. All the surviving patients are still under therapy.

\section{Discussion}

$\mathrm{RB}$ is the most common intraocular malignancy in infancy and childhood. Recently, more patients have achieved long-term survival. We searched PubMed, Ovid, and MEDLINE using the terms "RB, hepatic metastasis," "RB, liver metastasis," "RB, hepatic metastases," "RB, liver metastases," but no relevant 
articles were found. Only one article on metastatic RB mentioned cases of hepatic metastasis, but it did not include a detailed description and analysis. ${ }^{7}$ We describe a series of rare hepatic metastatic RB cases to provide pediatric oncologists and ophthalmological oncologists with a deeper understanding of the biological diversity of $\mathrm{RB}$, which is useful in the comprehensive evaluation of the patients.

The common symptoms of RB include leukocoria, strabismus, visual loss, ocular swelling, exophthalmos, etc. In this study, three patients had periorbital neoplasms, which corresponded to the severity and stage of the primary tumor. Only one patient with terminal-stage disease had hepatomegaly (Fig. 2).

As the liver receives an abundant double blood supply from the hepatic artery and portal vein, it is a common metastatic site for many malignant tumors. ${ }^{12}$ However, hepatic metastasis is rare in RB. In this study, all patients had severe disease with multiple system involvement, including the CNS, bone marrow, bone, and lymph nodes. CNS and bone metastases occurred in all the patients (Table 1). Therefore, we believe that hepatic metastasis from RB occurs in the advanced stage when several organs and systems are involved, including the liver. The liver is neither the only metastatic site nor a susceptible organ for RB.

High-risk pathological factors of RB include invasion of the optic nerve posterior to the ethmoid plate (including optic nerve stumps) and extensive invasions of the choroid, sclera, anterior chamber, iris, and ciliary body. ${ }^{13}$ These factors are related to a high risk for metastasis and dissemination. Only three of the patients in this study underwent enucleation, and each had extensive invasions of the choroid (Table 3). We infer that hepatic metastasis might occur in the course of spread through the choroid. However, due to our study's limited sample size, there is need for further evidence-based verification of the correlation between hepatic metastasis and extensive invasions of the choroid.

NSE is an important indicator of neurogenic tumors. ${ }^{14}$ In all five patients, NSE levels were elevated significantly at the time of hepatic metastasis diagnosis, which was consistent with distant metastasis. After chemotherapy, NSE levels decreased in four patients, while exophthalmos was relieved, and the periorbital neoplasm shrank. NSE levels remained high in one patient with end-stage disease, who died 1 month after diagnosis of hepatic metastasis. However, NSE levels are generally elevated in patients with distant metastasis; therefore, it could not be used as a diagnostic index for patients with RB showing hepatic metastases.

AFP is synthesized by the liver and is closely related to primary liver malignancies; however, AFP levels are often normal in patients with hepatic metastases. ${ }^{15}$ AFP was measured in three patients in this study, and the values were within the normal range. We also analyzed biochemical indicators of liver metabolism (Table 4). We found elevated LDH levels, but they could be associated with extensive metastasis and a high tumor burden. Considering the high sensitivity and poor specificity of LDH, it cannot be used as a tumor marker for hepatic metastasis from RB.

Imaging methods for diagnosing hepatic metastases include ultrasound, abdominal CT, and PET-CT. ${ }^{16-18}$ Imaging findings vary considerably among patients. They could involve low-density foci on CT (due to the lack of blood supply to metastases) and intratumoral necrosis. All the CT studies in this series showed hepatic metastases with low-density lesions; some were diffuse, while some were isolated, and some cases involved multiple foci, while others involved single foci. Calcification was obvious in the tumor in one patient and was even more obvious after treatment. This reminds us of the characteristic calcified plaques of intraocular lesions of RB; whether they are related or not needs further verification.

The prognosis of RB with distant metastasis is poor, especially when the CNS is involved. ${ }^{5,19-20}$ All the patients had CNS metastases, from which two died, one had disease progression, and the other two achieved partial remission and underwent active treatment. Considering CNS involvement, the prognosis is not good. However, in terms of hepatic metastases only, four of the five patients achieved partial remission after chemotherapy, while one showed no response to treatment. Two patients died, and one showed disease progression. The causes of death and disease progression were not hepatic metastases. Therefore, we speculate that chemotherapy is effective for hepatic metastases, and hepatic metastasis is not a direct cause of death. 
The prognosis of patients with RB having hepatic metastases is worse than that of patients with RB having other metastases, including metastases in the bone marrow, bone, and lymph nodes. However, this may be because hepatic metastasis occurs in the end-stage period and with CNS involvement. To an extent, this also confirms that CNS involvement is a very important prognostic factor.

To conclude, hepatic metastasis of RB is rare, occurs as distant metastases, and usually occurs with CNS and bone metastases. Systemic assessment is necessary in extensive metastasis, and abdominal CT can be helpful in screening for hepatic metastasis, which is characterized by low-density lesions with calcification. Presently, NSE, AFP, and LDH cannot be used as tumor markers for early detection of RB hepatic metastasis. In future, we need to look for appropriate indicators. Aggressive chemotherapy may be effective in controlling the progression of hepatic metastases, but the prognosis is poor, due to multiple organ metastases, especially CNS metastases. However, hepatic metastasis is not a direct cause of death. We should focus on looking for an effective treatment for RB with CNS metastases.

\section{Conflict of Interest Statement}

The authors declare that they have no conflicts of interest.

\section{Acknowledgments}

\section{Funding Sources}

This work was supported by "Climbing the Peak" Talent Plan of Beijing Hospital Authority [NO: DFL20180201].

\section{Author Contributions}

Huali Gu designed the study and wrote the initial draft of the manuscript. Yizhuo Wang and Dongsheng Huang contributed to the analysis and interpretation of data and assisted in the preparation of the manuscript. All other authors contributed to interpretation and critically reviewed the manuscript. All authors approved the final version of the manuscript and agree to be accountable for all aspects of the work. They also ensure that questions related to the accuracy or integrity of any part of the work are appropriately investigated and resolved.

\section{References}

1. Kivela, T. The epidemiological challenge of the most frequent eye cancer: retinoblastoma, an issue of birth and death. Br J Ophthalmol 2009;93:1129-1131.

https://doi.org/10.1136/bjo.2008.150292

2. Abramson D H, Schefler A C. Update on retinoblastoma. Retina 2005;24:828-848. https://doi.org/10.1097/00006982-200412000-00002

3. Khlopas A, Chughtai M, Elmallah R K, et al. Current concepts for diagnosis and treatment of retinoblastoma in Germany: aiming for safe tumor control and vision preservation. Klinische Pdiatrie 2012;224;339-347. https://doi.org/10.1055/s-0032-1327563

4. Dongsheng H, Yi Z, Weiling Z, et al. Study on clinical therapeutic effect including symptoms, eye preservation rate, and follow-up of 684 children with retinoblastoma. Eur J Ophthalmol 2013;23:532-538. https://doi.org/ 10.5301/ejo.5000245

5. Gunduz K, Muftuoglu O, Gunalp I, et al. Metastatic retinoblastoma clinical features, treatment, and prognosis. Ophthalmology 2006;113:1558-1566.

https://doi.org/10.1016/j.ophtha.2006.03.039

6. Radhakrishnan V, Kashyap S, Neelam Pushker M S, et al. Outcome, pathologic findings, and compliance in orbital retinoblastoma (International Retinoblastoma Staging System stage III) 
treated with neoadjuvant chemotherapy: a prospective study. Ophthalmology 2012;119:1470-1477. https://doi.org/10.1016/j.ophtha.2012.01.029

7. Dunkel IJ, Khakoo Y, Kernan NA, et al. Intensive multimodality therapy for patients with stage 4a metastatic retinoblastoma. Pediatr Blood Cancer 2010;55:55-59. https://doi.org/ 10.1002/pbc.22504

8. Dunkel IJ, Chan HSL, Jubran R, et al. High-dose chemotherapy with autologous hematopoietic stem cell rescue for stage 4B retinoblastoma. Pediatr Blood Cancer 2010;55:149-152. https://doi.org/ $10.1002 /$ pbc. 22491

9. $\mathrm{Hu} \mathrm{H}$, Zhang $\mathrm{W}$, Wang $\mathrm{Y}$, et al. Clinical features, treatment and prognosis of retinoblastoma in distant metastasis stage. [Zhong hua yan ke za zhi] Chinese Journal of Ophthalmology 2017;53:121-127. https://doi.org/10.3760/cma.j.issn.0412-4081.2017.02.010.

10. Cancela MB, Zugbi S, Winter U, et al. A decision process for drug discovery in retinoblastoma. Invest New Drugs 2020. [Online ahead of print]

https://doi.org/10.1007/s10637-020-01030-0

11. Trotti A, Colevas AD, Setser A, et al. CTCAE v3.0: development of a comprehensive grading system for the adverse effects of cancer treatment. Semin Radiat Oncol 2003;13:176-181.

https://doi.org/10.1016/S1053-4296(03)00031-6

12. Fernandez-Pineda I, Sandoval JA, Davidoff A M. Hepatic metastatic disease in pediatric and adolescent solid tumors. World J Hepatol 2015;7:1807-1817.

https://doi.org/10.4254/wjh.v7.i14.1807

13. Künkele, Annette, Wilm J, Holdt M, et al. Neoadjuvant/adjuvant treatment of high-risk retinoblastoma: a report from the German Retinoblastoma Referral Centre. Br J Ophthalmol 2015;99:949. https://doi.org/0.1136/bjophthalmol-2014-306222

14. Zhi-Ping L, Ke-Ying Z, Li-Li C, et al. A preliminary study of retinoblastoma-related serum tumor markers. Chinese Journal of Contemporary Pediatrics 2017;19:318-321. https://doi.org/10.7499/j.issn.10088830.2017.03.013

15. Abdel-Hamid NM, Abouzied MM, Nazmy MH, et al. A suggested guiding panel of seromarkers for efficient discrimination between primary and secondary human hepatocarcinoma. Tumor Biol 2016;37:2539-2546.

https://doi.org/10.1007/s13277-015-4025-7

16. Hakime A, Yevich S, Tselikas L, et al. Percutaneous thermal ablation with ultrasound guidance. Fusion imaging guidance to improve conspicuity of liver metastasis. Cardiovasc Intervent Radiol 2017;5:1-7. https://doi.org/10.1007/s00270-016-1561-5

17. Nan, Wang, Ye, et al. Differentiation of liver abscess from liver metastasis using dual-energy spectral CT quantitative parameters. Eur J Radiol 2019;4:204-208.

https://doi.org/10.1016/j.ejrad.2019.02.024

18. Tsurusaki M, Sofue K, Murakami. Current evidence for the diagnostic value of gadoxetic acid-enhanced magnetic resonance imaging for liver metastasis. Hep Res 2016;9:853-861. https://doi.org/10.1111/hepr.12646

19. Dimaras H, Kimani K, Dimba EA, et al. Retinoblastoma. Lancet 2012; 379:1436-1446. https://doi.org/ 10.1016/S0140-6736(11)61137-9

20. $\mathrm{Hu} \mathrm{H}$, Zhang $\mathrm{W}$, Wang $\mathrm{Y}$, et al. Characterization, treatment and prognosis of retinoblastoma with central nervous system metastasis. BMC Ophthalmology 2018;18:107. https://doi.org/10.1186/s12886-018-0772-8 


\section{Hosted file}

TABLE 1.pdf available at https://authorea.com/users/386270/articles/501752-clinical-featuresand-imaging-manifestations-of-retinoblastoma-with-hepatic-metastasis

\section{Hosted file}

TABLE 2.pdf available at https://authorea.com/users/386270/articles/501752-clinical-featuresand-imaging-manifestations-of-retinoblastoma-with-hepatic-metastasis

\section{Hosted file}

TABLE 3.pdf available at https://authorea.com/users/386270/articles/501752-clinical-featuresand-imaging-manifestations-of-retinoblastoma-with-hepatic-metastasis

\section{Hosted file}

TABLE 4.pdf available at https://authorea.com/users/386270/articles/501752-clinical-featuresand-imaging-manifestations-of-retinoblastoma-with-hepatic-metastasis

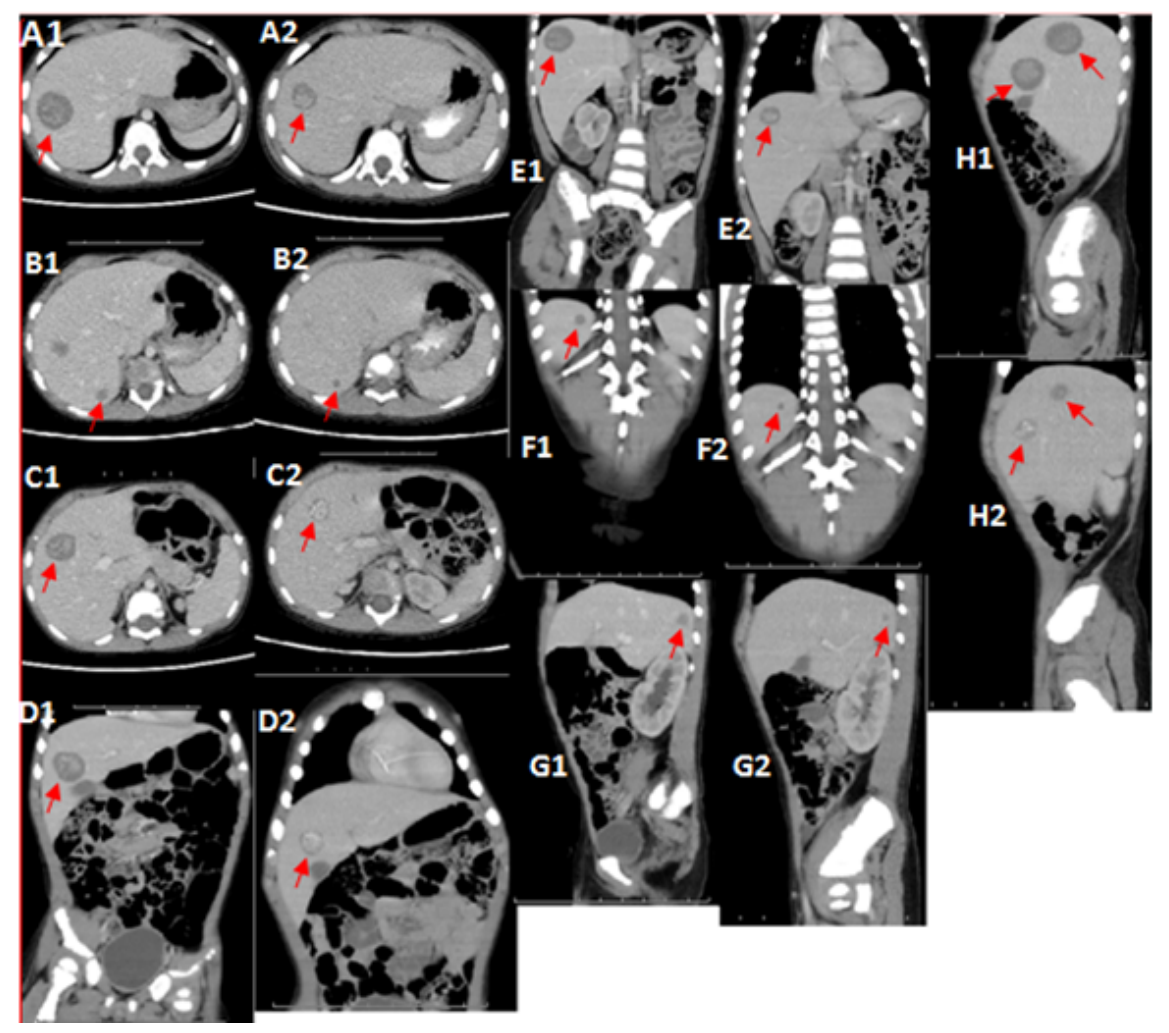




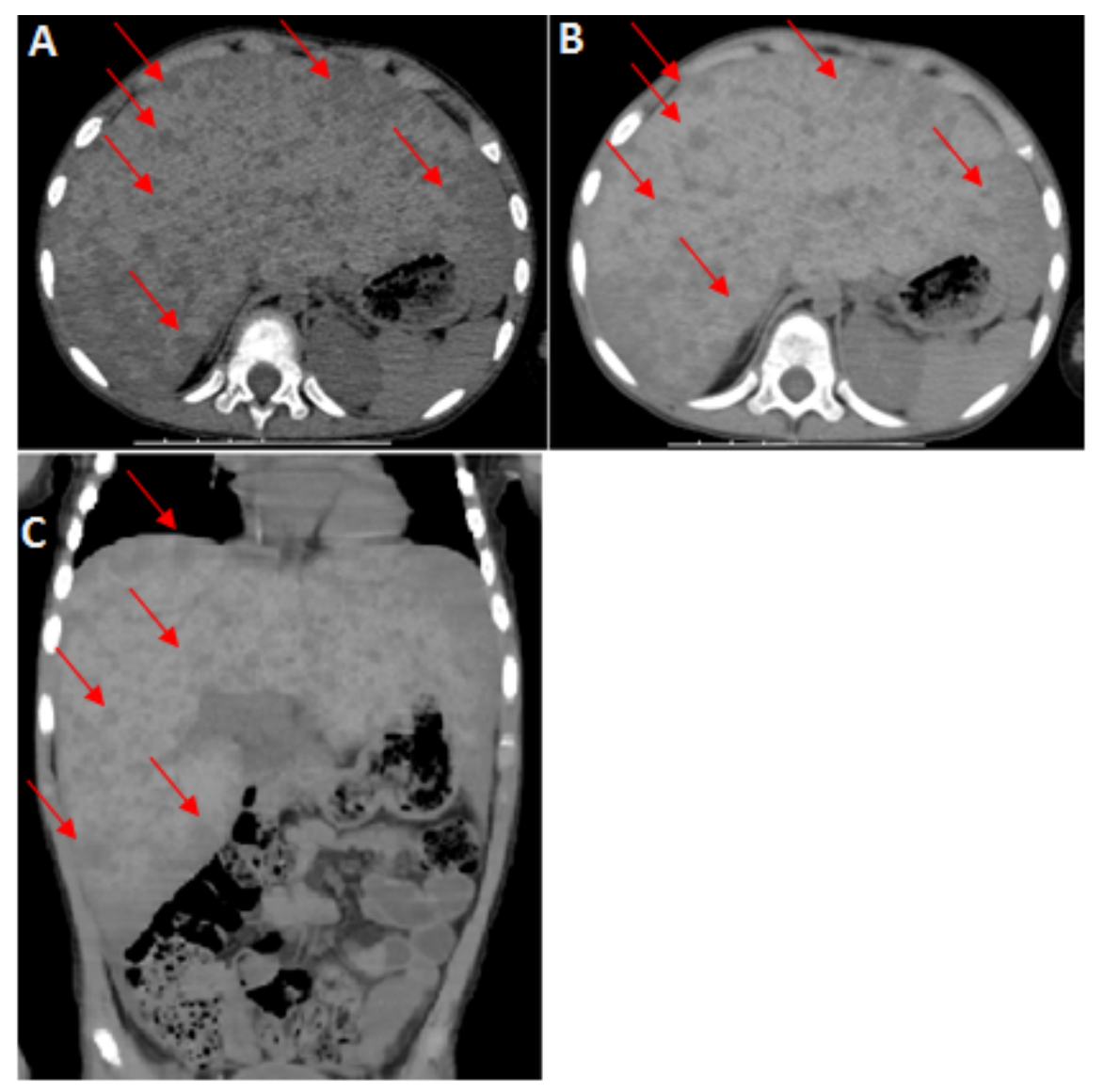

